\title{
Photonics with Multiwall Carbon Nanotube Arrays
}

\author{
Elefterios Lidorikis ${ }^{\dagger}{ }^{\dagger *}$ and Andrea C. Ferrari ${ }^{\ddagger}$
}

${ }^{\dagger}$ Department of Materials Science \& Engineering, University of loannina, loannina GR-45110 Greece, and ₹Engineering Department, Cambridge University, Cambridge, CB3 OFA U.K.

ABSTRACT We investigate the photonic properties of two-dimensional nanotube arrays for photon energies up to $40 \mathrm{eV}$ and unveil the physics of two distinct applications: deep-UV photonic crystals and total visible absorbers. We find three main regimes: for small intertube spacing of $20-30 \mathrm{~nm}$, we obtain strong Bragg scattering and photonic band gaps in the deep-UV range of $25 \sim 35 \mathrm{eV}$. For intermediate spacing of $40-100 \mathrm{~nm}$, the photonic bands anticross with the graphite plasmon bands resulting into a complex photonic structure, and a generally reduced Bragg scattering. For large spacing $>150 \mathrm{~nm}$, the Bragg gap moves into the visible and decreases due to absorption. This leads to nanotube arrays behaving as total optical absorbers. Our results can guide the design of photonic applications in the visible and deep UV ranges.

KEYWORDS: carbon nanotube · nanotube array · photonic crystal · effective medium theory · deep UV photonics · ultrablack materials

*Address correspondence to elidorik@cc.uoi.gr.

Received for review February 6, 2009 and accepted April 07, 2009.

Published online April 15, 2009. 10.1021/nn900123a CCC: $\$ 40.75$

C 2009 American Chemical Society waves when their period is comparable to the incident wavelength (Bragg scattering). If the scattering is strong enough, complete spectral gaps may open where light propagation is prohibited in all directions. These are called photonic band gaps. Many important effects and applications of photonic crystals, such as light localization, ${ }^{48}$ inhibition of spontaneous emission, ${ }^{49}$ light guidance and manipulation, ${ }^{50,51}$ and, more recently, superlensing ${ }^{52}$ and meta-materials exhibiting negative refraction, ${ }^{53,54}$ have been extensively studied both theoretically and experimentally. The materials typically used up to date have been semiconductors, ${ }^{51,55,56}$ polymers, ${ }^{57-59}$ and metals. ${ }^{60-63}$ In terms of CNT-based photonic crystals, however, little has been done. In particular, some evidence of Bragg diffraction has been reported, ${ }^{39-41}$ and an effective medium theory has been applied in the long wavelength limit. ${ }^{42,43}$ To the best of our knowledge no measurements or calculations have yet been performed proving the existence and properties of full photonic band gaps in periodic arrays of aligned CNTs. Here, we present full photonic band structure and reflectivity calculations and explore the CNT performance and potential applications in the technologically important area of photonic crystals.

CNTs consisting of a single rolled-up graphene layer are called single-wall carbon nanotubes (SWNTs), while multiwall carbon nanotubes (MWNT) have two or more walls. It is now possible to grow arrays of CNTs in defined positions, orientations and lengths, down to low temperatures. ${ }^{64-70}$ While SWNTs have diameters typically on the order of a nanometer, MWNTs can be controllably deposited and aligned with diameters up to hundreds nanometers. ${ }^{64-68}$ Bragg scattering 
occurs when the periodicity is comparable to the wavelength of light. For a given periodicity, this is maximized when the scatterer size is comparable to the spacing, that is, for volume filling ratios between $5 \%$ and $50 \%,{ }^{44}$ or, diameter/spacing ratios between 0.25 and 0.8 . Given the large range of achievable MWNT diameters, it is in principle expected that MWNT arrays may induce strong Bragg-like scattering for a wide frequency range between visible and UV, and thus may be ideal candidates for Vis-UV photonics.

An important feature, which defines the photonic properties of CNT arrays, is their dielectric response. This is anisotropic. ${ }^{2-15}$ However, apart from the cylindrical geometry, the general features observed are similar to those of bulk graphite. ${ }^{10-12,15}$ Given that CNTs are rolled-up graphene layers, this is somewhat expected: graphite is highly anisotropic, with different dielectric response functions $\varepsilon_{\|}(\omega)$ and $\varepsilon_{\perp}(\omega)$ depending on the electric field being polarized along or perpendicular to its c-axis. For a CNT, if the electric field is polarized parallel to its axis, it is also parallel to the graphite layers, otherwise both parallel and perpendicular contributions exist. Graphene, that is, a single graphite layer, has been shown to have a similar optical response to bulk graphite for light incident normal to its plane and polarized along it. ${ }^{71-74}$ Assuming curvature effects to be small and the local dielectric function to be well described by that of graphite, for electric field polarized parallel to the CNT axis we have only the $\varepsilon_{\perp}(\omega)$ contribution, while for perpendicular we need consider both $\varepsilon_{\perp}(\omega)$ and $\varepsilon_{\|}(\omega)$. Such "rolled-up" graphite was used to model CNTs electron energy loss spectra (EELS), ${ }^{75-78}$ and good agreement was found down to the SWNT scale. A "spherical" graphite model was also invoked to explain EELS of multishell fullerenes. ${ }^{77-82}$ We thus assume bulk graphite to be adequate for the dielectric function of MWNTs with more than 10 walls (diameters larger than $7 \mathrm{~nm}$ ). Indeed, graphene flakes with $>10$ layers are indistinguishable from graphite. ${ }^{71,83,84}$

\section{RESULTS AND DISCUSSION}

We study the photonic response of two-dimensional MWNT square arrays under transverse magnetic (TM) polarized illumination (i.e., MWNT infinitely long, with light polarized parallel to the CNT axis, and incident along a normal plane). In reality, of course, MWNT are not infinite, but, as long as their length is several times their diameter and the incident light wavelength, this approximation is valid. Since in photonic band gap applications the scatterer diameter must be comparable to the wavelength of light, the length/diameter ratios of more than 50 , which can be routinely produced in MWNT arrays, definitely satisfy these requirements. ${ }^{64-66} \mathrm{TM}$ is the optimal polarization for opening a photonic band gap in an array of dielectric cylindrical objects in vacuum. ${ }^{44}$ For this polarization, both square and hexagonal arrays are good candidates

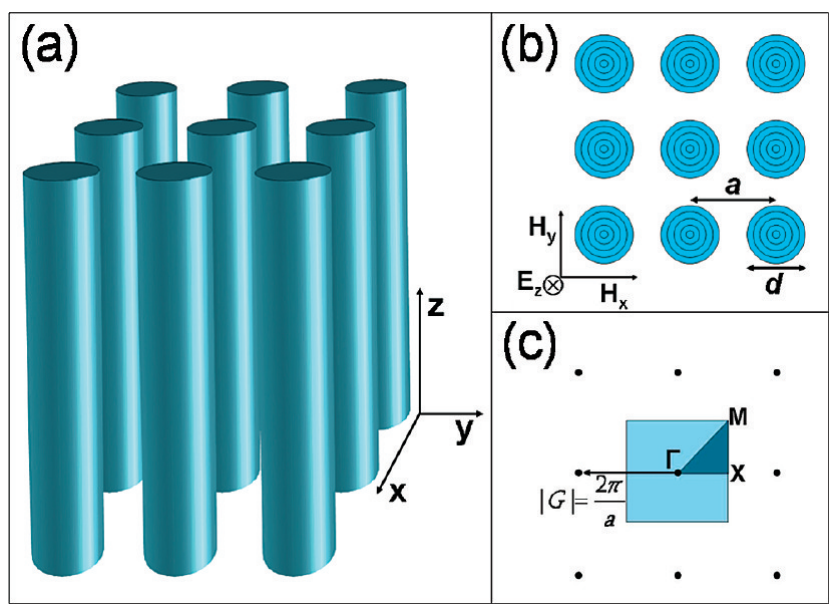

Figure 1. (a) Schematic of geometry: two dimensional square array of MWNTs with diameter $d$ and separation $a$. (b) Two dimensional projection of the system. We study the photonic properties in the TM polarization, i.e., for light polarized with electric field parallel to the tube axis. (c) Reciprocal lattice of structure in panel b: light blue shading is the first Brillouin zone, and dark blue shading is its irreducible part.

and yield similar qualitative results. ${ }^{44}$ Given its simplicity in setup and analysis, we here focus on the square array. Furthermore, for this polarization only $\varepsilon_{\perp}(\omega)$ is needed, that is, that best measured experimentally. ${ }^{85}$ Finally, we assume nanotubes with interior walls all the way to their center. A schematic of this arrangement and its 2D projection are shown in Figures $1 \mathrm{a}, \mathrm{b}$, respectively. The spatial periodicity of the MWNT array results into an equivalent periodicity in the reciprocal space, with a reciprocal lattice unit vector of magnitude $|G|=$ $2 \pi / a$, with $a$ the inter-MWNT lattice spacing, Figure $1 c$. The reciprocal lattice primitive cell is the first Brillouin zone, shown in light blue shading in Figure 1c, while in dark blue shading is the irreducible part of the first Brillouin zone, revealing the independent photon wavevectors and their symmetry. The most important,

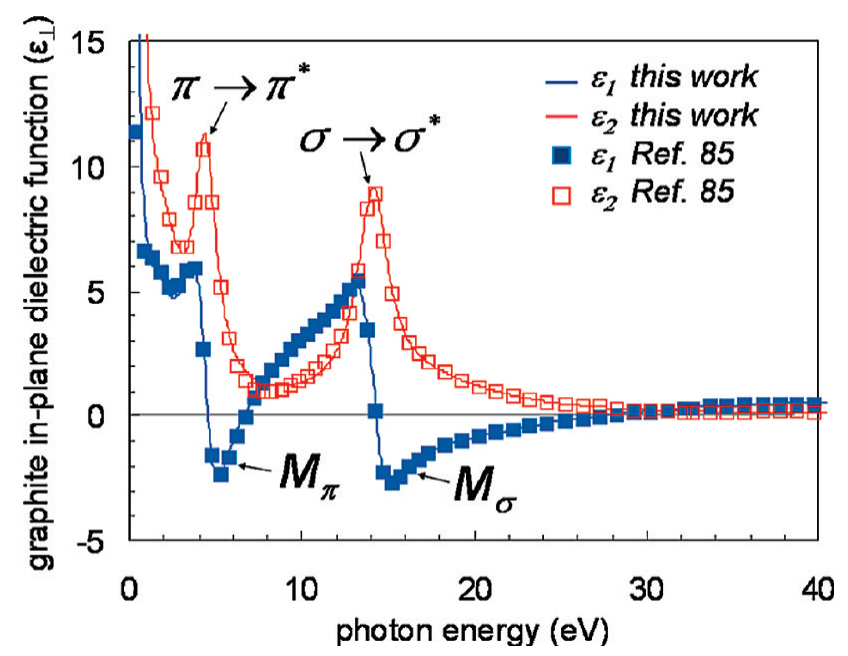

Figure 2. Dielectric function of graphite for the $E \perp c$ polarization. Solid lines represent the model used in this work, while symbols indicate the model used in ref 85 . 
(a)
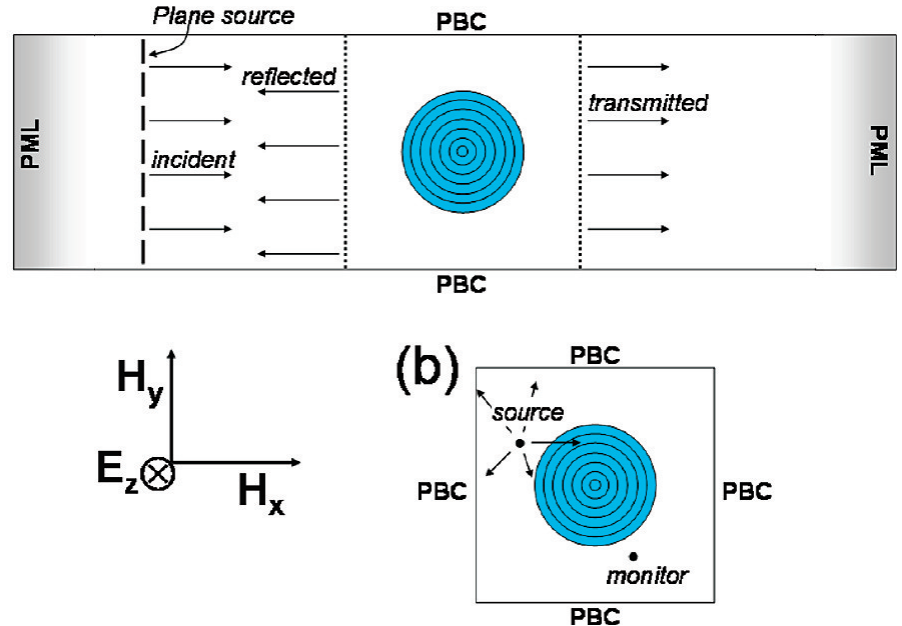

Figure 3. Computational cell for (a) transmission and reflection calculation; periodic boundary conditions on the side, perfectly matched-layer (absorbing) boundary conditions at the ends. A wide-spectrum plane source is used. (b) Band structure calculation; periodic boundary conditions all around the unit cell. A wide spectrum point-source and a monitor point are used, both on nonsymmetric points of the unit cell. In both cases the computational discretization is $1 \mathrm{~nm} /$ grid.

in terms of band structure and gap formation, are the ones tracing its periphery along $\Gamma \rightarrow \mathrm{X} \rightarrow \mathrm{M} \rightarrow \Gamma^{44}$

We use the graphite in-plane dielectric function $\varepsilon_{\perp}(\omega)$ proposed by ref 85 (see also refs $86-88$ ), which is a compilation of data from several groups, consistent with continuity and Kramers-Kronig requirements. This can be fitted with a Drude-Lorentz model: ${ }^{89}$

$$
\varepsilon_{\perp}(\omega)=1-\frac{\omega_{\mathrm{p}}^{2}}{\omega^{2}+i \omega / \tau}+\sum_{m=1}^{M} \frac{\sigma_{m}^{2}}{\omega_{m}^{2}-\omega^{2}-i \omega \gamma_{m}}(1)
$$

where the first term is the Drude free electron contribution, the second contains Lorentz oscillators corresponding to interband transitions. $\omega_{p}$ and $\tau$ are free electron plasma frequency and relaxation time, $\hbar \omega_{m}$ $\sigma_{m}$, and $\gamma_{m}$ are transition energy, oscillator strength, and decay rate for the Lorentz terms. To accurately reproduce the experimental graphite dielectric function we treat these as fit parameters. Reference 85 reported an excellent fit to the experimental data using $M=7$. However, they also had frequency-dependent decay rates

TABLE 1. Parameters Used for Our Graphite Dielectric Function Fit Shown in Figure $2^{a}$

\begin{tabular}{cccc}
$\boldsymbol{m}$ & $\boldsymbol{\gamma}_{\boldsymbol{m}}$ & $\boldsymbol{\omega}_{\boldsymbol{m}}$ & $\boldsymbol{\sigma}_{\boldsymbol{m}}$ \\
\hline 0 & 2.32 & 0.00 & 2.09 \\
1 & 1.58 & 0.74 & 4.32 \\
2 & 2.59 & 2.38 & 5.19 \\
3 & 1.60 & 4.46 & 8.25 \\
4 & 1.01 & 14.42 & 3.61 \\
5 & 2.17 & 14.12 & 14.63 \\
6 & 9.94 & 16.36 & 14.97 \\
7 & 8.40 & 21.45 & 6.05
\end{tabular}

${ }^{a}$ All numbers are in eV. The Drude term is for $m=0$, where $\gamma_{0} \equiv 1 / \tau$ and $\sigma_{0} \equiv$ $\omega_{p}$. $\gamma_{m}(\omega)$, which is incompatible with our numerical method. Thus, we perform a new fit with static decay rates, using again $M=7$. Our model parameters are shown in Table 1, while Figure 2 plots our model dielectric function $\varepsilon_{\perp}(\omega)$ and that of ref 85 , showing that they are almost identical.

The important features to note in the $\varepsilon_{\perp}$ of the graphite are, in the imaginary part, the two absorption peaks due to the $\pi \rightarrow \pi^{*}$ transition at $\sim 4.5$ $\mathrm{eV}$ and the $\sigma \rightarrow \sigma^{*}$ at $\sim 14.3 \mathrm{eV}$. In $\operatorname{Re}\left\{\varepsilon_{\perp}\right\}$ these are accompanied by metallic bands (i.e., energy regions with negative $\operatorname{Re}\left\{\varepsilon_{\perp}\right\}^{89}$ ) occurring just above those transitions, indicated in Figure 2 by $M_{\pi}, M_{\sigma}$, which terminate at plasma energies of $\sim 7$ and 27 $\mathrm{eV}$, respectively ${ }^{85}$ (where $\operatorname{Re}\left\{\varepsilon_{\perp}\right\}=0$ ). For low energies both real and imaginary part increase, indicating large absorption, while at high energies the response asymptotically approaches zero with $\operatorname{Re}\left\{\varepsilon_{\perp}\right\} \rightarrow 1$ and $\operatorname{Im}\left\{\varepsilon_{\perp}\right\} \rightarrow 0$.

We use the finite-difference time-domain (FDTD) method ${ }^{90-92}$ to calculate (1) reflectivity and effective medium properties, and (2) photonic band structure. For the former we solve the transmission problem of a single array row, as shown in Figure $3 a$ (where the CNT is periodically repeated in the vertical direction because of the periodic boundary condition). For the photonic band structure, the computational cell consists of a single unit cell, as shown in Figure $3 \mathrm{~b}$, where the CNT is periodically repeated in both vertical and horizontal directions because of the periodic boundary conditions (i.e., we simulate a "bulk" $2 \mathrm{D}$ array). More details on these calculations are given in the Methods section.

The onset of multiple scattering effects is determined by the value of a. This happens for $\lambda \approx 2 a\langle n\rangle$. We first calculate the response of a square array with $a$ $=20 \mathrm{~nm}$ and surface filling ration $f=35 \%$ (i.e., diameter $d=13.5 \mathrm{~nm}$ ). At this spacing, Bragg scattering is expected around $\lambda \approx 35 \mathrm{~nm}(\sim 35 \mathrm{eV})$. This puts us close to the edge of the dielectric-function model range (see Figure 2) but fairly far from the two plasmons, so absorption will be minimal and thus there will be higher chances to observe multiple scattering. The chosen $f$ is typical to promote Bragg scattering effects. If the values are too large absorption dominates (for $f>50 \%$ ), while if too small the scattering cross sections diminishes (for $f<5 \%$ ).

Figure 4 plots the calculated effective material constants for this system. In the long wavelength limit $\lambda$ $\gg a$ (e.g., $\lambda>60 \mathrm{~nm}$ or $\hbar \omega<20 \mathrm{eV}$ ), the effective permittivity is very similar to that of bulk graphite up to some scaling factor. This means that no resonant effects develop and the effective response is merely a volume average of the dielectric function of the individual constituents, ${ }^{42}$ that is, MWNT and vacuum. At the Bragg resonance, $\hbar \omega \approx 33 \mathrm{eV}$ (or $\lambda \approx 37 \mathrm{~nm}$ ), on the other hand, a strong effective permittivity and mag- 


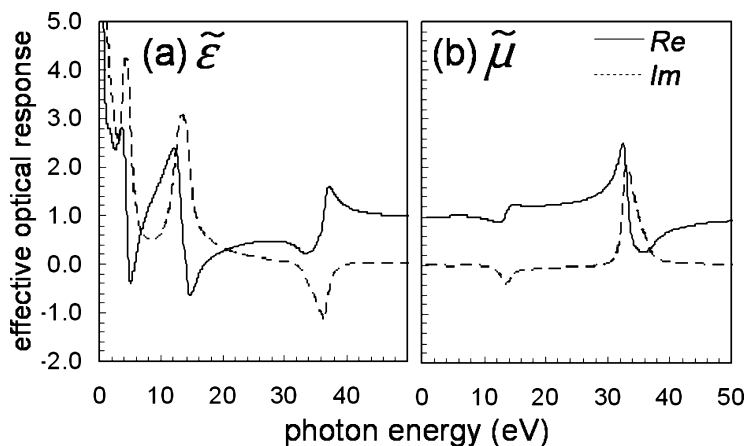

Figure 4. Effective medium parameters for the $a=20 \mathrm{~nm}, f$ $=35 \%$ square array of MWNTs.

netic response develop. These are characteristic of photonic crystals inside the first band gap, ${ }^{93}$ that is, how an effective uniform medium incorporates multiple reflections occurring in the discrete system at the Bragg condition. This is a signature of strong Bragg-like multiple scattering in deep UV.

With these effective material parameters we calculate the expected response of any number of MWNT array rows. Figure $5 \mathrm{c}-\mathrm{e}$ plot reflection, absorption, and transmission from 10 rows at $a=20 \mathrm{~nm}$ and $f=35 \%$. This amounts to a total system length of $200 \mathrm{~nm}$, Figure $5 \mathrm{a}$. For comparison we also plot the response of a solid graphite film, with thickness chosen to have the same density per unit cross section, that is, $200 \mathrm{~nm} \times$
$0.35=70 \mathrm{~nm}$, Figure 5b. This ensures that light will effectively "cover" the same amount of graphitic material for both systems, thus serving as a "normalized" comparison between the two responses.

The major difference is at the Bragg reflectivity peak, which is totally absent in the bulk case. This is accompanied by a strong dip in the absorption and transmission. On the other hand, both systems have metallic peaks in the reflectivity and dips in absorption $\left(M_{\pi}\right.$ and $M_{\sigma}$ in Figure $\left.5 c-e\right)$, with the bulk graphite response being more pronounced. This is expected since for these energies the array behaves as bulk, being in the subwavelength limit $\lambda \gg a$. Indeed, Figure $4 a$ shows that, while the effective dielectric constant $\tilde{\varepsilon}$ is still metallic (i.e., negative), it is however less pronounced, thus allowing more light to penetrate. This gives less reflection and more absorption. In general, far from the Bragg peak, the array is a better absorber than a reflector. However the transmission is much more suppressed for the array system, close to 2 orders of magnitude at the $\pi \rightarrow \pi^{*}$ transition energy and 5 orders of magnitude at the $\sigma \rightarrow \sigma^{*}$ transition energy. The array system, thus, promotes stronger absorption.

To explore the structural parameter space, we vary $a$, with $f=35 \%$. Figure 6 a plots the semi-infinite reflection curves, each shifted by 0.25 for clarity. As $a$ increases, the Bragg peak shifts to lower energies, and an-

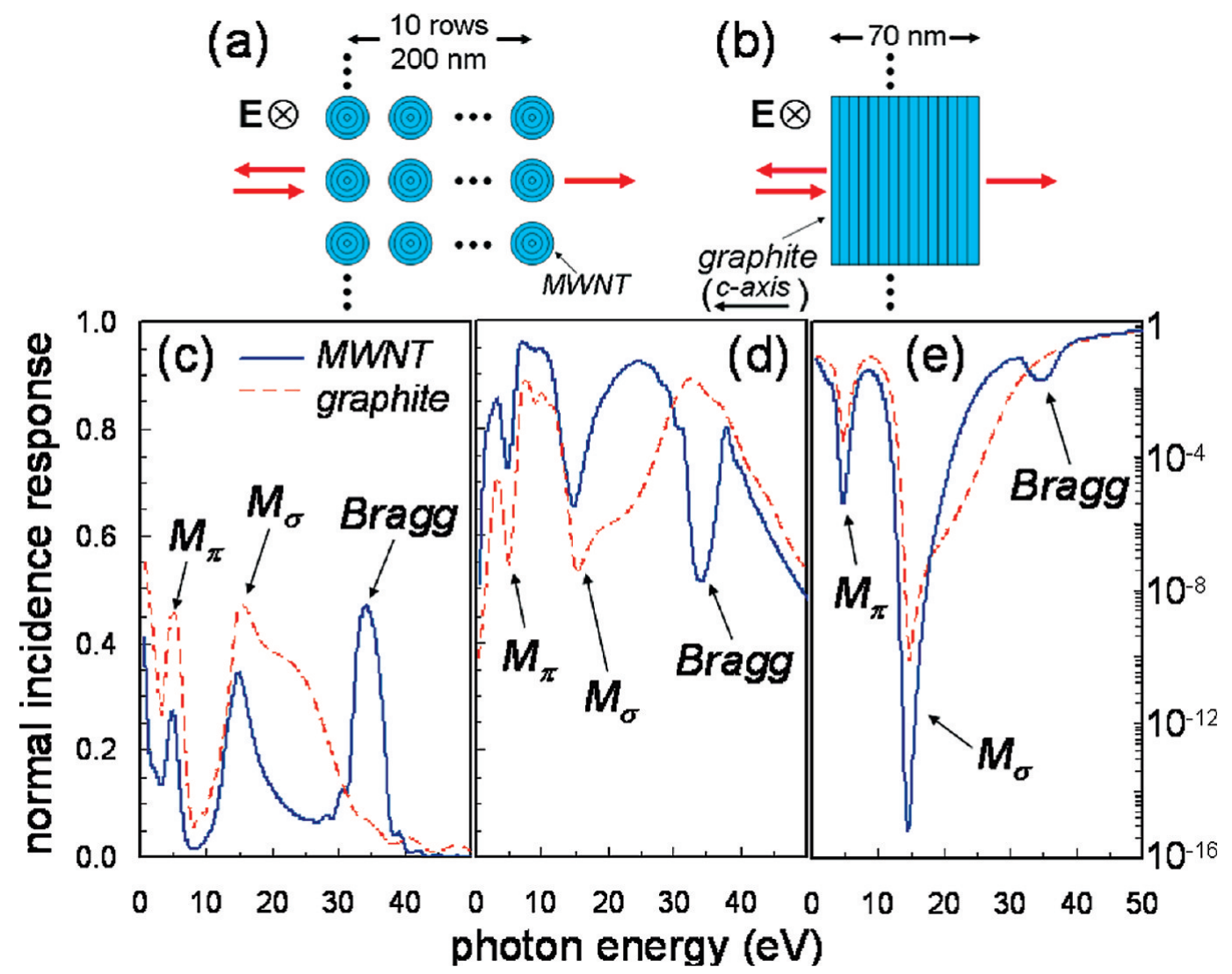

Figure 5. (a) Scheme of a 10-row MWNT system; (b) scheme of a 70nm bulk graphite system; (c-e) response of 10 rows MWNT array with $\alpha=20 \mathrm{~nm}$ and $f=35 \%$. The dotted line is the response of $70 \mathrm{~nm}$ thick graphite: (c) reflection, (d) absorption, and (e) transmission. 


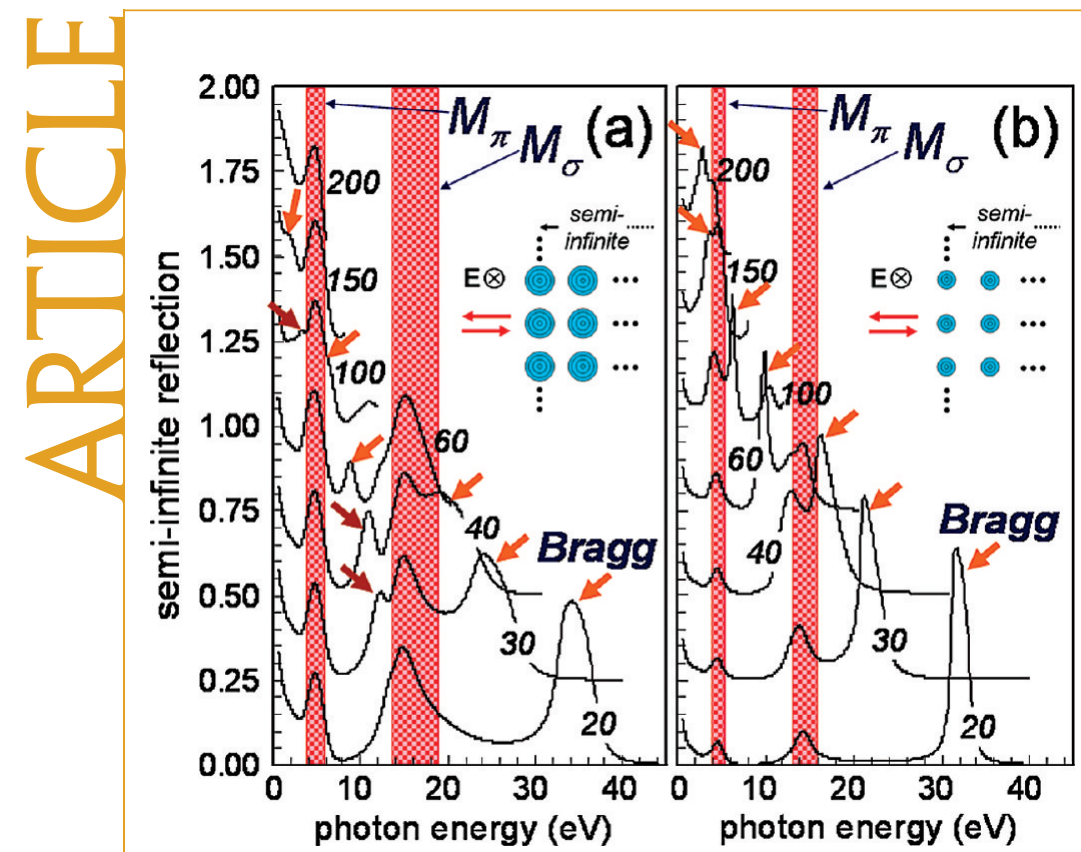

Figure 6. Semi-infinite reflection curves for various lattice constants and (a) $f$ $=35 \% ;(b) f=10 \%$. Each terminates at the diffraction limit $\lambda=a$. Shaded vertical bars mark the two metallic bands, whose position is independent from the spacing. Orange arrows point out the Bragg peaks, while brown arrows point out the new Bragg peaks appearing below the metallic bands due to anticrossing.

ticrosses the $M_{\sigma}$ metallic band. At $a=30 \mathrm{~nm}$ this causes a new peak to appear, just below $M_{\sigma}$ and at $a=40$ $\mathrm{nm}$ the new peak is much more pronounced, while the original Bragg peak merges with $M_{\sigma}$. One way to understand this is that, as the Bragg condition shifts to lower energies, the dielectric constant changes and, thus, the condition self-consistently readjusts to a new energy. In some cases the Bragg condition gets partially destroyed and resatisfied at a lower energy, appearing as if the Bragg peak splits in two. At even larger $a$, the Bragg peak moves between $M_{\pi}$ and $M_{\sigma}$, but is less pronounced because of increased absorption. Above $a=$ $100 \mathrm{~nm}$, it crosses $M_{\pi}$ and moves into the visible. There, it gets smeared out, because of absorption. Note that for all $a, M_{\pi}$ and $M_{\sigma}$ remain at the same energy.

The response evolution is not so pronounced if we assume a smaller $f=10 \%$, Figure $6 \mathrm{~b}$ (for the smallest

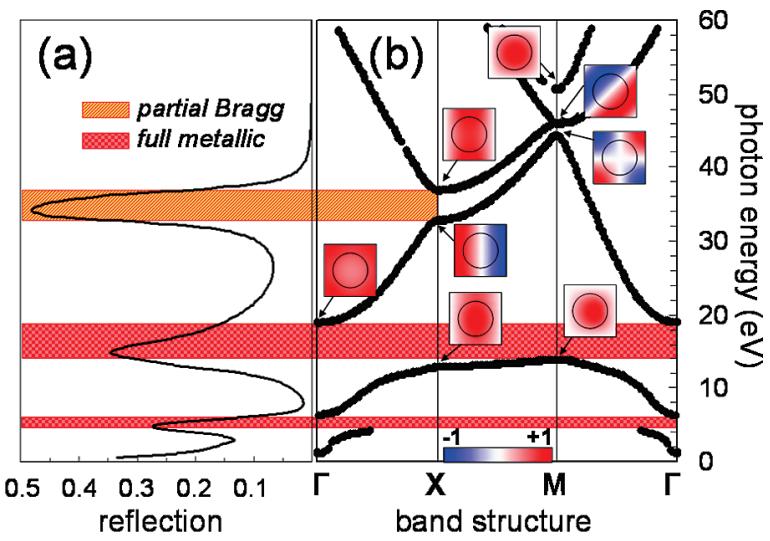

Figure 7. (a) Semi-infinite reflectivity and (b) photonic band structure for $a=20 \mathrm{~nm} ; f=35 \% ; d=13.5 \mathrm{~nm}$. $a=20 \mathrm{~nm}$ this implies a diameter $d=7 \mathrm{~nm}$ ). We observe a narrower and taller Bragg peak and, at the same time, narrower but shorter metallic peaks. As the Bragg peak shifts to lower energies for increasing separations, it does not significantly anticross the metallic bands. In particular, only for $a=40 \mathrm{~nm}$ do we see a small repulsion between the two peaks, still not strong enough to produce the splitting observed for $f=35 \%$. This is expected because both bands are very narrow, with a much smaller overlap. In most cases this gives much smaller interaction, except for $a=40 \mathrm{~nm}$, where both bands have the same energy.

To elucidate the Bragg- $M_{\sigma}$ anticrossing for the $f=$ $35 \%$ case, we calculate the band structure for $a=20$ and $40 \mathrm{~nm}$ in Figures $7 \mathrm{~b}$ and $8 \mathrm{~b}$. These also plot the corresponding normal incidence semi-infinite reflection, for direct comparison. The red bands mark the metallic gaps, orange marks the partial Bragg gaps, while the yellow band in Figure 8b marks a full Bragg gap. We associate every peak in the reflectivity with the gaps in the band structure. For $a=20 \mathrm{~nm}$, we only have a partial Bragg gap, and all three gaps (Bragg, $M_{\sigma}$, and $M_{\pi}$ ) are well separated. The photonic band structure around the metallic gaps results from the anticrossing of the free photon band and the localized tube modes below the $\pi \rightarrow \pi^{*}$ or $\sigma \rightarrow \sigma^{*}$ transitions (where the dielectric constant has large positive values), and is similar to what observed in $2 \mathrm{D}$ arrays of polaritonic materials in the THz regime. ${ }^{92}$ For $a=40 \mathrm{~nm}$, the band structure is more complex. The Bragg gap evolves into a full band gap, while a new propagating mode appears below $M_{\sigma}$ (where, as discussed above, $\operatorname{Re}\left\{\varepsilon_{\perp}\right\}$ has large positive values), resulting into a new partial Bragg gap. This complex behavior resulting from the band anticrossing could be combined with measurements to become a valuable diagnostic tool of dielectric function and geometric structure of MWNTs arrays.

The photonic eigenmodes at high-symmetry points are shown as insets in Figures $7 \mathrm{~b}$, and $8 \mathrm{~b}$. In Figure 7b we separate two different regions: below $30 \mathrm{eV}$ the modes are localized resonances supported by the high dielectric constant (i.e., below the $\pi \rightarrow \pi^{*}$ and $\sigma \rightarrow \sigma^{*}$ transitions). Above $30 \mathrm{eV}$ the modes resemble the free photon bands with a weak scattering perturbation. The only difference from what usually is observed in $2 \mathrm{D}$ photonic crystals ${ }^{44}$ is that, since here $\operatorname{Re}\left\{\varepsilon_{\perp}\right\}<1$ (see Figure 2), the order in increasing energy in which the modes appear is reversed, that is the $s$-type monopole mode appears at higher energy than the $p$-type dipole. In Figure $8 \mathrm{~b}$ the free photon bands move downward and anticross the localized modes, resulting into a new branch and partial Bragg gap at $\sim 12 \mathrm{eV}$. The modes in either side of this gap are similar, but in energy reverse order from those at the top gap of $20 \mathrm{eV}$, since here $\operatorname{Re}\left\{\varepsilon_{\perp}\right\}>1$. These modal patterns may also be useful to study surface and impurity states in MWNT arrays. ${ }^{44}$ 
Figure 6 indicates that, apart from the inter-MWNT spacing, the photonic response is also dependent on the surface-filling ratio. Given that this is a very important experimental parameter, we explore it more in Figure $9 a$, where the reflection from 5 array rows at fixed $a=20 \mathrm{~nm}$ is plotted for seven different $f$. The main trend is, as expected, that reflection increases for increasing $f$ : For fixed spacing, increasing $f$ amounts to increasing the MWNT diameter and, thus, the scattering cross section. There is a difference, however, between the two metallic peaks and the Bragg peak. While the metallic peak position does not shift in energy and monotonously increases in magnitude, the Bragg peak blue-shifts and reaches a maximum at $f \approx 35 \%$, after which it decreases. The blue-shift is explained from the fact that, since the Bragg peak appears at $\lambda \approx 2 a\langle n\rangle$, and given that at these energies the tube refractive index is less than 1 (because $\operatorname{Re}\left\{\varepsilon_{\perp}\right\}<1$ ), increasing diameter decreases the average index and thus blue-shifts the response. The nonmonotonic response at large $f$ is because absorption increases due to increased material coverage, and thus, Bragg scattering reduces. At even larger $f$, scatterers start to overlap, further reducing the response, which eventually equates the bulk graphite reflectivity at the $f \rightarrow 100 \%$ limit.

It is even more interesting to see how the response is modified when we remove the finite thickness restriction, that is, look at the semi-infinite reflection for different filling ratios, as plotted in Figure 9b. The metallic peak behavior of the semi-infinite system is hardly different from before, from which we conclude that the system's response already saturates at five rows. Thus, at these energies the system behaves effectively as a metal with a small penetration depth. In contrast, the Bragg peak has maximum reflectivity at the smallest $f$, with the peak magnitude monotonically decreasing for increasing $f$. The blue-shift is the same as in the 5-row case, but in addition we notice a strong decrease in bandwidth for the smaller $f$. The reason is that the structure is now semi-infinite and, thus, there are enough array rows for the Bragg backscattering mechanism to build-up, even for the smallest $f$. In this case the only remaining limiting factor is absorption: the smaller $f$ has less absorption and, thus, the maximum peak reflection, reaching above $70 \%$ as $f$ goes to zero. This, however, is only true for energies exactly inside the corresponding Bragg gap. Since this decreases with $f$, so does the reflection peak bandwidth.

Thus, the optimal surface filling ratio $f_{\max }$ is different depending on the number of array rows. Figure 10a plots the calculated peak reflection for $a=20 \mathrm{~nm}$ for different number of rows as a function of $f$. Indeed, depending on the number of rows, the peak reflection reaches a specific maximum as a function of $f$. This moves to smaller $f_{\max }$ for increasing array rows. This trend is shown in Figure 10b, which plots the maximum reflection and tube diameter corresponding to

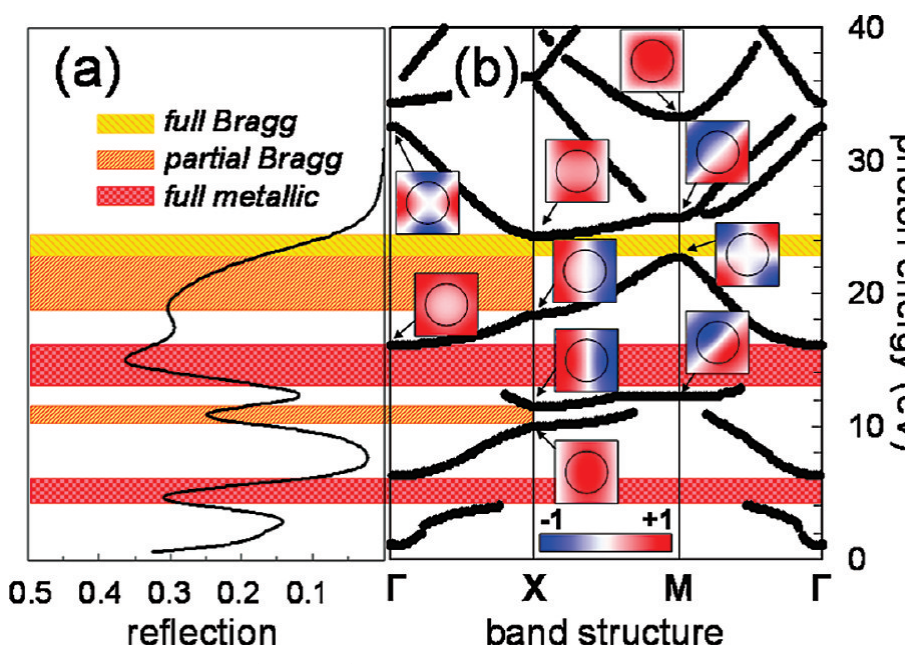

Figure 8. (a) Semi-infinite reflectivity and (b) photonic band structure for $a$ $=40 \mathrm{~nm} ; f=35 \% ; d=27 \mathrm{~nm}$.

$f_{\max }$ as a function of the number of array rows. Thus, we can tailor the photonic response of MWNT arrays in the deep UV range. These results may find application in emerging UV technologies such as extreme UV lithography. ${ }^{94-97}$

Thus far we focused on the UV spectrum, i.e. a small enough so that the Bragg peak is far from the metallic bands. This is because, as shown in Figure 6, for increasing $a$ the Bragg peak intermixes with the metallic bands and diminishes in strength. We now turn to the other side of the spectrum, at $a$ large enough so that the Bragg peak appears at a much lower energy than the $\pi \rightarrow \pi^{*}$ peak, that is, optical frequencies. This is the region where most proposals for CNT-based photonic crystals have been reported. ${ }^{38-41}$ The dielectric function there (inset of Figure 11) is characterized by very large absorption: a moderately large real part $\varepsilon_{1} \equiv$
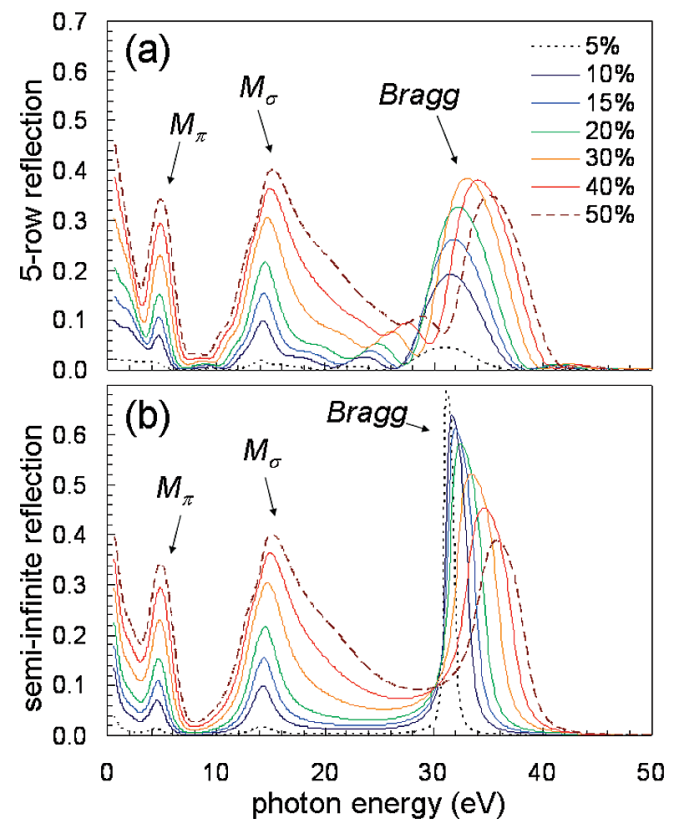

Figure 9. Normal reflection for $a=20 \mathrm{~nm}$ and varying $f$ : (a) five array rows, (b) semi-infinite. 


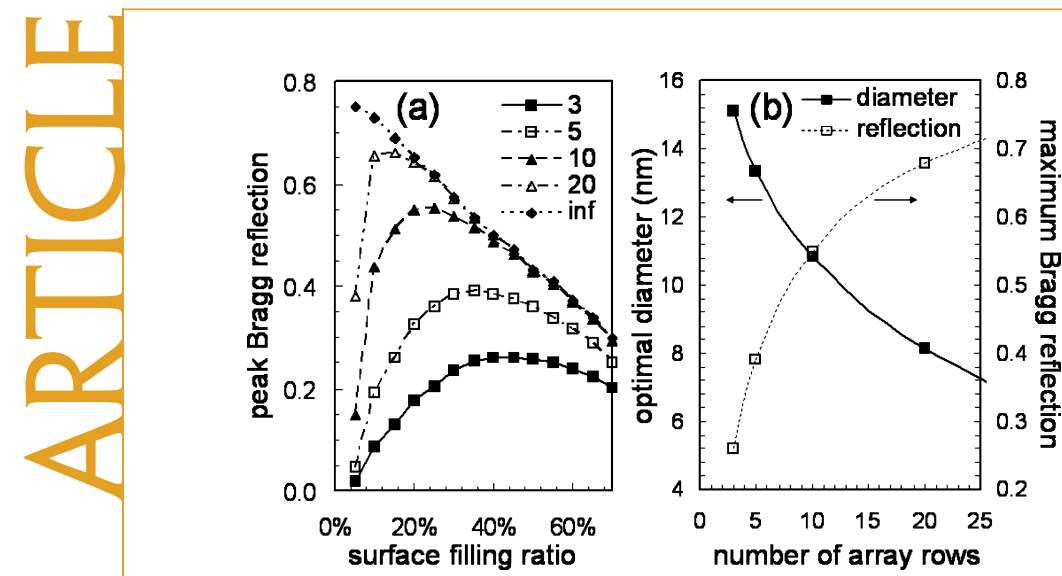

Figure 10. (a) Peak Bragg reflection for the $a=20 \mathrm{~nm}$ array as a function of $f$ for five systems with different number of array rows. (b) Optimal diameter and maximum achieved Bragg reflection as a function of the number of rows used for the $a=20 \mathrm{~nm}$ array.

$\operatorname{Re}\left\{\varepsilon_{\perp}\right\}$ accompanied by a much larger imaginary part $\varepsilon_{2} \equiv \operatorname{Im}\left\{\varepsilon_{\perp}\right\}$. Figure 11a shows the semi-infinite normalincidence reflection when $a=500 \mathrm{~nm}, d=113 \mathrm{~nm}$, and $f=4 \%$. These parameters are very close to those proposed by refs 38-41 and typical for aligned MWNT arrays. $^{64-66}$ We do observe Bragg peaks, the fundamental reaching up to $50 \%$. The higher order Bragg peaks are smaller and narrower, as usually expected for photonic crystals. ${ }^{44}$ While not significantly lower than Figure 9 for $a=20 \mathrm{~nm}$ and $f=5 \%$, this is not particularly encouraging, given the variety of semiconducting materials and versatile processing technology that can yield photonic crystals at these frequencies. ${ }^{55,56}$ Thus, we do not expect pristine MWNT arrays to play a competitive role in applications requiring visible light reflectors and/or modulators. As pointed out above, this is due to the large absorption of graphite at optical frequencies, which limits Bragg backscattering. On the other hand, coated MWNTs ${ }^{98-100}$ or MWNTs encapsulating nanowires, ${ }^{101}$ having a different dielectric func-

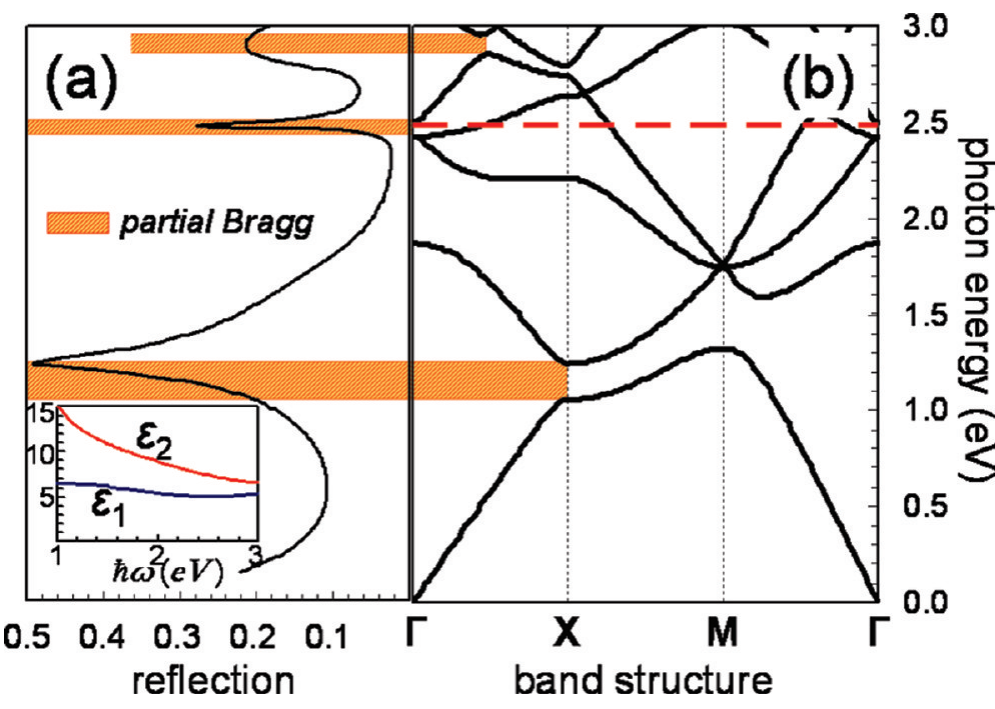

Figure 11. (a) Semi-infinite reflectivity and (b) photonic band structure for $a=500$ $\mathrm{nm} ; f=4 \% ; d=113 \mathrm{~nm}$. In the inset, the in-plane graphite dielectric function for visible photon energies is shown. The band structure in $b$ is calculated neglecting absorption. tion, could play a significant role in this frequency region.

For completeness, Figure $11 \mathrm{~b}$ also plots the corresponding band structure of a nonabsorbing material with $\epsilon=5.25$ (due to its extreme value, it is not possible to obtain a meaningful photonic band structure including the absorption). The peaks in reflection match well with the partial gaps in the band structure. The dashed red line in Figure $11 \mathrm{~b}$ marks the diffraction limit $a=\lambda$.

We further explore the MWNT array response by calculating the reflection and transmission at different $f$, keeping $a=500 \mathrm{~nm}$. Figure 12a plots the total scattering (reflection + transmission) for 10 array rows (i.e., a film thickness of $5 \mu \mathrm{m}$ ). We note that very quickly the Bragg peaks diminish, they are not visible already at $f=$ $8 \%$. At $f=50 \%$ the response is featureless and very close to bulk graphite (dashed line in Figure 12a). This is in marked contrast to the $a=20$ case, where the Bragg peaks were persistent even at large $f \approx 50 \%$. In between the two extremes of Figure 12a, however, there is a very interesting region where the scattering just below the diffraction limit (i.e., a little below $2.5 \mathrm{eV}$ ) drops to very low values. In particular, for $f=12 \%$ there is a photon energy for which total scattering goes to $\sim 7 \times 10^{-5}$, or inversely, the absorption goes above $99.99 \%$. At this point, the incident wave couples strongly, via second order Bragg scattering, to guided resonances within the array. Reflections get minimized, and as the guided resonances are almost parallel to the array surface, they get totally absorbed. Above the $a=\lambda$ diffraction limit, on the other hand, diffraction in vacuum becomes allowed, that is, a new reflection mode becomes available, and thus the overall reflection sharply increases.

In Figure $12 \mathrm{~b}$ we plot the absorption of the 10-row, $a=500 \mathrm{~nm}, f=12 \%$ case, for energies close to the absorption resonance. The background is color-coded for illustration purposes. Most of the green part of the spectrum, between 512-570 $\mathrm{nm}$, has absorption $>99 \%$, peaking at $>99.99 \%$ for $\lambda=532 \mathrm{~nm}$. This makes MWNT arrays an extremely dark material. ${ }^{31,32,102-107}$ Such materials have important applications in solar collectors, ${ }^{108}$ optical measurement systems, ${ }^{104}$ etc. The record holder in terms of darkness is a recently reported low density MWNT system. ${ }^{31}$ It is interesting that while both systems, ours and that of ref 31, are nanotube-based, they utilize a different mechanism: here we propose that second order Bragg scattering resonantly redirects the incident light in the film so it gets quickly absorbed, while ref 31 uses a low density MWNT array to index-match the vacuum and thus achieve minimum reflectance. Unavoidable in the latter approach, however, is that total absorption requires thicker films: ${ }^{32}$ our estimated absorption 
coefficient is $\alpha \approx 1 \mu \mathrm{m}^{-1}$, about 1 order of magnitude larger than the $\alpha=0.12 \mu \mathrm{m}^{-1}$ of ref 31, making our thickness requirement an order of magnitude smaller. Furthermore, our normal-incidence reflectance is around $\sim 5 \times 10^{-5}$, almost an order of magnitude less than ref 31 . On the other hand, ref 31 has a wider angular and wavelength acceptance. A complete comparison between the two methods requires additional calculations for p-polarization, variable-angle in-plane and out-of-plane incidence, roughened film surfaces, etc., beyond the scope of this work.

We believe our results to be quite general. Small discrepancies between the actual dielectric function of a given MWNT and the graphite model used here will not alter the main qualitative features we have obtained, that is, the existence of strong Bragg multiple scattering in deep UV frequencies, or strong absorption at optical frequencies. This is also not expected to qualitatively vary under structural changes, that is, other MWNT arrangements such as hexagonal or small structural disorder (in radius, position, and alignment), which may only slightly reduce the size and effectiveness of the band gaps. We can thus use the results and methodologies presented here in conjunction with experiments to (a) assess the nanotube dielectric function from the fine differences in response, and (b) assist in designing new nanotube-based photonic applications such as UV mirrors, waveguides, and lenses, or efficient optical absorbers. On the other hand, we can use our calculations to assess the features of a given MWNT film from its reflectance response, such as MWNT alignment, arrangement, spacing, diameter, disorder, etc.

In conclusion, we calculated the expected response of MWNT arrays under TM illumination, assuming the dielectric response of graphite. We find that strong Bragg scattering with full photonic band gaps can be observed at UV energies. Typical size and structure parameters for this are $20-30 \mathrm{~nm}$ nanotube spacing and $12-20 \mathrm{~nm}$ diameters. These are within the range of
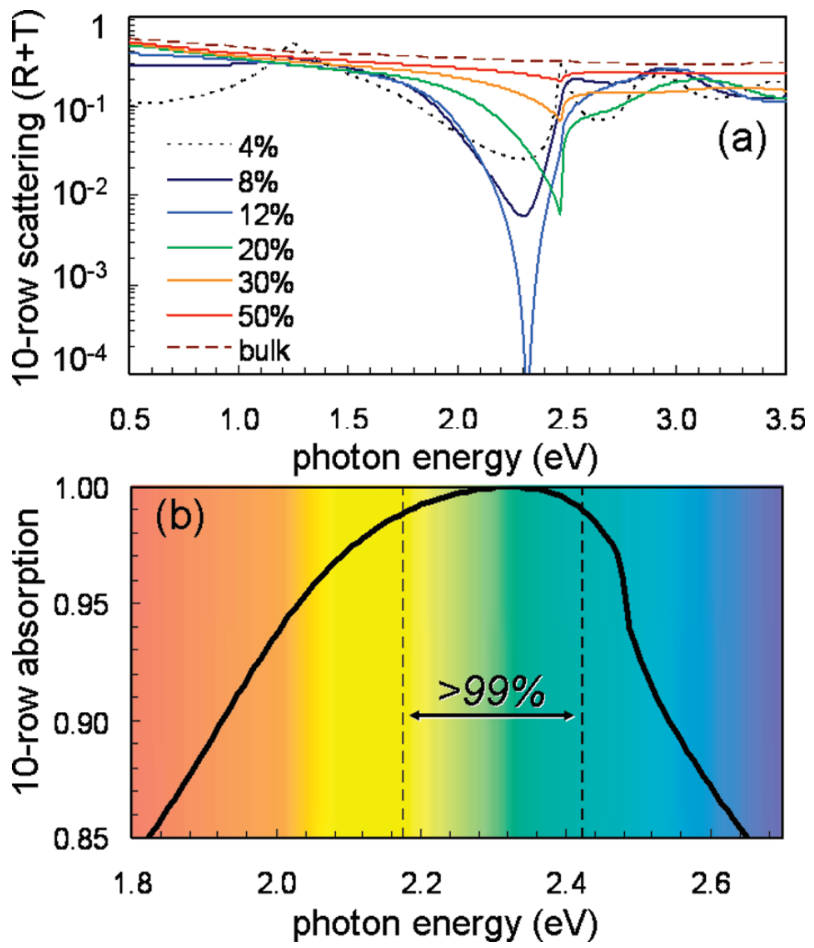

Figure 12. (a) Normal scattering (reflection + transmission) from 10 rows: $a=500 \mathrm{~nm}$ and different $f$. The dashed line marks bulk graphite reflectivity. (b) Normal absorption from 10 rows: $a=500$ $\mathrm{nm} ; f=12 \%$. Total absorption ( $>99.99 \%$ ) is achieved for $\lambda=532$ $\mathrm{nm}$, while the bandwidth of larger than $99 \%$ absorption extends through most of the green spectrum.

what can be achieved today, making nanotubes a natural candidate for deep UV photonics. At larger spacing, the Bragg condition red-shifts and gets modulated by the two graphite plasmons resulting into a more complicated response involving band anticrossing and splitting. Finally, at inter-MWNT spacings of the order of $150 \mathrm{~nm}$ or more, the Bragg peak shifts to optical frequencies, where the system is more suitable for total absorption. These results will help in designing future nanotube-based Vis-UV photonic applications.

\section{METHODS}

To calculate the photonic response we use the finitedifference time-domain (FDTD) method, ${ }^{90}$ in which Maxwell's equations are solved on a computational grid,

$$
\nabla \times \mathbf{E}=-\mu \partial_{\mathrm{t}} \mathbf{H}, \quad \nabla \times \mathbf{H}=\varepsilon_{0} \partial_{t} \mathbf{E}+\partial_{t} \mathbf{P}_{0}+\sum_{m=1}^{M} \partial_{t} \mathbf{P}_{m}
$$

where material polarization is taken into account through the polarizabilities $\vec{P}$

$$
\partial_{t}{ }^{2} \mathbf{P}_{0}+\partial_{t} \mathbf{P}_{0} / \tau=\omega_{p}{ }^{2} \varepsilon_{0} \mathbf{E}, \quad \partial_{t}{ }^{2} \mathbf{P}_{m}+\gamma_{m} \partial_{t} \mathbf{P}_{m}+\omega_{m}{ }^{2} \mathbf{P}_{m}=\sigma_{m}^{2} \varepsilon_{0} \mathbf{E}
$$

We calculate (i) reflectivity and effective medium properties and (ii) photonic band structure. For the former we start by solving the transmission problem of a single array row, as shown in
Figure 3a (where the CNT is periodically repeated in the vertical direction because of the periodic boundary condition). Two calculations are done, one with and one without tubes, and the complex transmitted and reflected amplitudes $t$ and $r$ (at the dotted lines) are obtained by taking the appropriate ratios. As long as we remain below the diffraction limit, we can use these to calculate the effective medium quantities as follows: ${ }^{91}$ from $t, r$ we get the corresponding transfer matrix $M=[t-r \cdot r / t, r / t ;-r / t, 1 / t]$, which we then evaluate in the semi-infinite layer limit $M^{\infty}=$ $\lim _{n \rightarrow \infty} M^{n}$ (usually $\left.n \approx 10^{4}\right)$. This gives us the semi-infinite reflection $r_{\infty}=-M_{21}^{\infty} / M_{22}^{\infty}$, which in turn is related through the Fresnel equations to an effective material impedance $\tilde{z}=\left(r_{\infty}\right.$ $+1) /\left(r_{\infty}-1\right)$. The trace of the transfer matrix is related to an effective material index $\tilde{n}: \operatorname{Tr}\{M\}=2 \cos (\tilde{n} \omega a / c)$, where $a$ is the inter-MWNT spacing. From these we get the effective permittivity and permeability $\tilde{\varepsilon}(\omega)=\tilde{n}(\omega) / \tilde{z}(\omega)$ and $\tilde{\mu}(\omega)=$ $\tilde{n}(\omega) \cdot \tilde{z}(\omega)$, which we use to analytically calculate the reflection and transmission from any number of MWNT array rows. 
For the photonic band structure, the computational cell consists of a single unit cell, as shown in Figure $3 \mathrm{~b}$, where the CNT is periodically repeated in both vertical and horizontal directions because of the periodic boundary conditions (i.e., we simulate a "bulk" 2D array). Bloch periodic boundary conditions are applied corresponding to a particular wavevector $\mathbf{k}$ along the perimeter of the irreducible Brillouin zone. A point source and monitor are placed in low-symmetry points of the cell. The point source emits a broadband spectrum and we record the time series of the electric field values at the monitor. The peaks of the Fouriertransformed electric field correspond to the eigenfrequencies of the particular $\mathbf{k}$. Repeating for all k's we obtain the photonic band structure. ${ }^{92}$

Acknowledgment. We thank N. Marzari and B. Kozinsky for useful discussions. All calculations where performed at the Research Center for Scientific Simulations (RCSS) at the University of loannina. A.C.F. acknowledges funding from The Royal Society Brian Mercer Award for Innovation and the European Research Grant NANOPOTS.

\section{REFERENCES AND NOTES}

1. Jorio, A., Dresselhaus, M. S., Dresselhaus, G., Eds. Carbon Nanotubes: Advanced Topics in the Synthesis, Structures, Properties, and Applications; Springer: New York, 2008.

2. deHeer, W. A.; Bacsa, W. S.; Châtelain, A.; Gerfin, T.; Humphrey-Baker, R.; Forro, L.; Ugarte, T. Aligned Carbon Nanotube Films: Production and Optical and Electronic Properties. Science 1995, 268, 845-847.

3. Bubke, K.; Gnewuch, H.; Hempstead, M.; Hammer, J.; Green, M. L. H. Optical Anisotropy of Dispersed Carbon Nanotubes Induced by an Electric Field. Appl. Phys. Lett. 1997, 71, 1906-1908.

4. Rao, A. M.; Jorio, A.; Pimenta, M. A.; Dantas, M. S. S.; Saito, R.; Dresselhaus, G.; Dresselhaus, M. S. Polarized Raman Study of Aligned Multiwalled Carbon Nanotubes. Phys. Rev. Lett. 2000, 84, 1820-1823.

5. Sfeir, M. Y.; Wang, F.; Huang, L.; Chuang, C.-C.; Hone, J.; O'Brien, S. P.; Heinz, T. F.; Brus, L. E. Probing Electronic Transitions in Individual Carbon Nanotubes by Rayleigh Scattering. Science 2004, 306, 1540-1543.

6. Murakami, Y.; Chiashi, S.; Miyauchi, Y.; Hu, M.; Ogura, M.; Okubo, T.; Maruyama, S. Growth of Vertically Aligned Single-Walled Carbon Nanotube Films on Quartz Substrates and Their Optical Anisotropy. Chem. Phys. Lett. 2004, 385, 298-303.

7. Fry, D.; Langhorst, B.; Wang, H.; Becker, M. L.; Bauer, B. J.; Grulke, E. A.; Hobbie, E. K. Rheo-optical Studies of Carbon Nanotube Suspensions. J. Chem. Phys. 2006, 124, 054703.

8. Fagan, J. A.; Simpson, J. R.; Landi, B. J.; Richter, L. J.; Mandelbaum, l.; Bajpai, V.; Ho, D. L.; Raffaelle, R.; Hight Walker, A. R.; Bauer, B. J.; Hobbie, E. K. Dielectric Response of Aligned Semiconducting Single-Wall Nanotubes. Phys. Rev. Lett. 2007, 98, 147402.

9. Shoji, S.; Suzuki, H.; Zaccaria, R. P.; Sekkat, Z.; Kawata, S. Optical Polarizer Made of Uniaxially Aligned Short SingleWall Carbon Nanotubes Embedded in a Polymer Film. Phys. Rev. B 2008, 77, 153407.

10. Murakami, Y.; Einarsson, E.; Edamura, T.; Maruyama, S. Polarization Dependence of the Optical Absorption of Single-Walled Carbon Nanotubes. Phys. Rev. Lett. 2005, 94 , 087402.

11. Lin, M. F.; Shyu, F. L.; Chen, R. B. Optical Properties of WellAligned Multiwalled Carbon Nanotube Bundles. Phys. Rev. B 2000, 61, 14114-14118.

12. Pan, H.; Feng, Y.; Lin, J. Ab Initio Study of Electronic and Optical Properties of Multiwall Carbon Nanotube Structures Made up of a Single Rolled-up Graphite Sheet. Phys. Rev. B 2005, 72, 085415.

13. Kozinsky, B.; Marzari, N. Static Dielectric Properties of Carbon Nanotubes from First Principles. Phys. Rev. Lett. 2006, 96, 166801.
14. Marinopoulos, A. G.; Reining, L.; Olevano, V.; Rubio, A.; Pichler, T.; Liu, X.; Knupfer, M.; Fink, J. Anisotropy and Interplane Interactions in the Dielectric Response of Graphite. Phys. Rev. Lett. 2002, 89, 076402.

15. Guo, G. Y.; Chu, K. C.; Wang, D.-S.; Duan, C.-G. Linear and Nonlinear Optical Properties of Carbon Nanotubes from First-Principles Calculations. Phys. Rev. B 2004, 69, 205416.

16. Xie, R. H.; Jiang, J. Nonlinear Optical Properties of Armchair Nanotube. Appl. Phys. Lett. 1997, 71, 1029-1031.

17. Sun, X.; Yu, R. Q.; Xu, G. Q.; Hor, T. S. A.; Ji, W. Broadband Optical Limiting with Multiwalled Carbon Nanotubes. Appl. Phys. Lett. 1998, 73, 3632-3634.

18. Maeda, A.; Matsumoto, S.; Kishida, H.; Takenobu, T.; Iwasa, Y.; Shiraishi, M.; Ata, M.; Okamoto, H. Large Optical Nonlinearity of Semiconducting Single-Walled Carbon Nanotubes under Resonant Excitations. Phys. Rev. Lett. 2005, 94, 047404.

19. Liu, X.; Si, J.; Chang, B.; Xu, G.; Yang, Q.; Pan, Z.; Xie, S.; Ye, P.; Fan, J.; Wan, M. Third-Order Optical Nonlinearity of the Carbon Nanotubes. Appl. Phys. Lett. 1999, 74, 164-166.

20. Chen, Y.-C.; Raravikar, N. R.; Schadler, L. S.; Ajayan, P. M.; Zhao, Y.-P.; Lu, T.-M.; Wang, G.-C.; Zhang, X.-C. Ultrafast Optical Switching Properties of Single-Wall Carbon Nanotube Polymer Composites at $1.55 \mu \mathrm{m}$. Appl. Phys. Lett. 2002, 81, 975-977.

21. Zhao, J.; Chen, X.; Xie, J. R. H. Optical Properties and Photonic Devices of Doped Carbon Nanotubes. Anal. Chim. Acta 2006, 568, 161-170.

22. Yamashita, S.; Inoue, Y.; Maruyama, S.; Murakami, Y.; Yaguchi, H.; Jablonski, M.; Set, S. Y. Saturable Absorbers Incorporating Carbon Nanotubes Directly Synthesized onto Substrates and Fibers and Their Application to Mode-Locked Fiber Lasers. Opt. Lett. 2004, 29, 1581-1583.

23. Nakazawa, M.; Nakahara, S.; Hirooka, T.; Yoshida, M.; Kaino, T.; Komatsu, K. Polymer Saturable Absorber Materials in the $1.5 \mu \mathrm{m}$ Band Using Poly-methyl-methacrylate and Polystyrene with Single-Wall Carbon Nanotubes and Their Application to a Femtosecond Laser. Opt. Lett. 2006, 31, 915-917.

24. Rozhin, A. G.; Sakakibara, Y.; Namiki, S.; Tokumoto, M.; Kataura, H.; Achiba, Y. Sub-200-fs Pulsed Erbium-Doped Fiber Laser Using a Carbon Nanotube-Polyvinylalcohol Mode Locker. Appl. Phys. Lett. 2006, 88, 051118.

25. Della Valle, G.; Osellame, R.; Galzerano, G.; Chiodo, N.; Cerullo, G.; Laporta, P.; Svelto, O.; Morgner, U.; Rozhin, A. G.; Scardaci, V.; Ferrari, A. C. Passive Mode Locking by Carbon Nanotubes in a Femtosecond Laser Written Waveguide Laser. Appl. Phys. Lett. 2006, 89, 231115.

26. Scardaci, V.; Rozhin, A. G.; Hennrich, F.; Milne, W. I.; Ferrari, A. C. Carbon Nanotube-Polymer Composites for Photonic Devices. Phys. E 2007, 37, 115-118.

27. Sun, Z.; Rozhin, A. G.; Wang, F.; Scardaci, V.; Milne, W. I.; White, I. H.; Hennrich, F.; Ferrari, A. C. L-Band Ultrafast Fiber Laser Mode Locked by Carbon Nanotubes. Appl. Phys. Lett. 2008, 93, 061114.

28. Schmidt, A.; Rivier, S.; Steinmeyer, G.; Yim, J. H.; Cho, W. B.; Lee, S.; Rotermund, F.; Pujol, M. C.; Mateos, X.; Aguiló, M. Passive Mode Locking of Yb:KLuW Using a Single-Walled Carbon Nanotube Saturable Absorber. Opt. Lett. 2008, 33, 729-731.

29. Solodyankin, M. A.; Obraztsova, E. D.; Lobach, A. S.; Chernov, A. I.; Tausenev, A. V.; Konov, V. I.; Dianov, E. M. Mode-Locked $1.93 \mu \mathrm{m}$ Thulium Fiber Laser with a Carbon Nanotube Absorber. Opt. Lett. 2008, 33, 1336-1338.

30. Wang, F.; Rozhin, A. G.; Scardaci, V.; Sun, Z.; Hennrich, F.; White, I. H.; Milne, W. I.; Ferrari, A. C. Wideband-Tuneable, Nanotube Mode-Locked, Fibre Laser. Nat. Nanotechnol. 2008, 3, 738-742.

31. Yang, Z.-P.; Ci, L.; Bur, J. A.; Lin, S.-Y.; Ajayan, P. M. Experimental Observation of an Extremely Dark Material Made By a Low-Density Nanotube Array. Nano Lett. 2008, 8, 446-451.

32. Garcia-Vidal, F. J. Metamaterials: Towards the Dark Side. Nat. Photonics 2008, 2, 215-216. 
33. Ajiki, H.; Ando, T. Aharonov-Bohm Effect in Carbon Nanotubes. Phys. B 1994, 201, 349-352.

34. Wang, Y.; Kempa, K.; Kimball, B.; Carlson, J. B.; Benham, G.; Li, W. Z.; Kempa, T.; Rybczynski, J.; Herczynski, A.; Ren, Z. F. Receiving and Transmitting Light-Like Radio Waves: Antenna Effect in Arrays of Aligned Carbon Nanotubes. Appl. Phys. Lett. 2004, 85, 2607-2609.

35. Itkis, M. E.; Borondics, F.; Yu, A.; Haddon, R. C. Bolometric Infrared Photoresponse of Suspended Single-Walled Carbon Nanotube Films. Science 2006, 312, 413-416.

36. Misewich, J. A.; Martel, R.; Avouris, P.; Tsang, J. C.; Heinze, S.; Tersoff, J. Electrically Induced Optical Emission from a Carbon Nanotube FET. Science 2003, 300, 783-786.

37. Chen, J.; Perebeinos, V.; Freitag, M.; Tsang, J.; Fu, Q.; Liu, J.; Avouris, P. Bright Infrared Emission from Electrically Induced Excitons in Carbon Nanotubes. Science 2008, 310, 1171-1174.

38. Wang, Y.; Wang, X.; Rybczynski, J.; Wang, D. Z.; Kempa, K.; Ren, Z. F. Triangular Lattice of Carbon Nanotube Arrays for Negative Index of Refraction and Subwavelength Lensing Effect. Appl. Phys. Lett. 2005, 86, 153120.

39. Kempa, K.; Kimball, B.; Rybczynski, J.; Huang, Z. P.; Wu, P. F.; Steeves, D.; Sennett, M.; Giersig, M.; Rao, D.V.G.L.N.; Carnahan, D. L. Photonic Crystals Based on Periodic Arrays of Aligned Carbon Nanotubes. Nano Lett. 2003, 3, 13-18.

40. Rybczynski, J.; Kempa, K.; Wang, Y.; Ren, Z. F.; Carlson, J. B.; Kimball, B. R.; Benham, G. Visible Light Diffraction Studies on Periodically Aligned Arrays of Carbon Nanotubes: Experimental and Theoretical Comparison. Appl. Phys. Lett. 2006, 88, 203122.

41. Wu, P.; Kimball, B.; Carlson, J.; Rao, D.V.G.L.N. Light Scattering of Periodic $\beta$-Aligned Carbon Nanotubes. Phys. Rev. Lett. 2004, 93, 013902.

42. García-Vidal, F. J.; Pitarke, J. M.; Pendry, J. B. Effective Medium Theory of the Optical Properties of Aligned Carbon Nanotubes. Phys. Rev. Lett. 1997, 78, 4289-4292.

43. Reyes, E.; Krokhin, A. A.; Roberts, J. Effective dielectric constants of photonic crystal of aligned anisotropic cylinders and the optical response of a periodic array of carbon nanotubes. Phys. Rev. B 2005, 72, 155118.

44. Joannopoulos, J. D.; Meade, R. D.; Winn, J. N. Photonic Crystals; Princeton: New York, 1995.

45. Soukoulis, C. M., Ed. Photonic Crystals and Light Localization in the 21st Century; Kluwer Academic Publishers: Dordrecht, The Netherlands, 2001.

46. Joannopoulos, J. D.; Villeneuve, P. R.; Fan, S. H. Photonic Crystals: Putting a New Twist on Light. Nature (London) 1997, 386, 143-149.

47. López, C. Materials Aspects of Photonic Crystals. Adv. Mater. 2003, 15, 1679-1704.

48. John, S. Strong Localization of Photons in Certain Disordered Dielectric Superlattices. Phys. Rev. Lett. 1987, 58, 2486-2489.

49. Yablonovitch, E. Inhibited Spontaneous Emission in SolidState Physics and Electronics. Phys. Rev. Lett. 1987, 58 , 2059-2062.

50. Johnson, S. G.; Mekis, A.; Fan, S. H.; Joannopoulos, J. D. Molding the Flow of Light. Comput. Sci. Eng. 2001, 3, 3847.

51. Talneau, A.; Le Gouezigou, L.; Bouadma, N.; Kafesaki, M.; Soukoulis, C. M.; Agio, M. Photonic-Crystal Ultrashort Bends with Improved Transmission and Low Reflection at 1.55 um. Appl. Phys. Lett. 2002, 80, 547-549.

52. Luo, C.; Johnson, S. G.; Joannopoulos, J. D.; Pendry, J. B. All-Angle Negative Refraction without Negative Effective Index. Phys. Rev. B 2002, 65, 201104

53. Soukoulis, C. M.; Linden, S.; Wegener, M. Negative Refractive Index at Optical Wavelengths. Science 2007, 315, 47-49.

54. Smith, D. R.; Pendry, J. B.; Wiltshire, M. C. K. Metamaterials and Negative Refractive Index. Science 2004, 305, 788-792.

55. Vlasov, Y. A.; O’Boyle, M.; Hamann, H. F.; McNab, S. J. Active Control of Slow Light on a Chip with Photonic Crystal Waveguides. Nature (London) 2005, 438, 65-69.
56. Qi, M. H.; Lidorikis, E.; Rakich, P. T.; Johnson, S. G.; Joannopoulos, J. D.; Ippen, E. P.; Smith, H. I. A ThreeDimensional Optical Photonic Crystal with Designed Point Defects. Nature (London) 2004, 429, 538-542.

57. Campbell, M.; Sharp, D. N.; Harrison, M. T.; Denning, R. G.; Turberfield, A. J. Fabrication of photonic crystals for the visible spectrum by holographic lithography. Nature (London) 2000, 404, 53-56.

58. Urbas, A. M.; Maldovan, M.; DeRege, P.; Thomas, E. L. Bicontinuous Cubic Block Copolymer Photonic Crystals. Adv. Mater. 2002, 14, 1850-1853.

59. Xia, Y. N.; Gates, B.; Yin, Y. D.; Lu, Y. Monodispersed Colloidal Spheres: Old Materials with New Applications. Adv. Mater. 2000, 12, 693-713.

60. Fan, S. H.; Villeneuve, P. R.; Joannopoulos, J. D. Large Omnidirectional Band Gaps in Metallodielectric Photonic Crystals. Phys. Rev. B 1996, 54, 11245-11251.

61. Yannopapas, V.; Modinos, A.; Stefanou, N. Optical Properties of Metallodielectric Photonic Crystals. Phys. Rev. B 1999, 60, 5359-5365.

62. Fleming, J. C.; Lin, S. Y.; El-Kady, I.; Biswas, R.; Ho, K. M. AllMetallic Three-Dimensional Photonic Crystals with a Large Infrared Bandgap. Nature (London) 2002, 417, 52-55.

63. Shelby, R. A.; Smith, D. R.; Schultz, S. Experimental Verification of a Negative Index of Refraction. Science 2001, 292, 77-79.

64. Ren, Z. F.; Huang, Z. P.; Xu, J. W.; Wang, J. H.; Bush, P.; Siegal, M. P.; Provencio, P. N. Synthesis of Large Arrays of Well-Aligned Carbon Nanotubes on Glass. Science 1998, 282, 1105-1107.

65. Chhowalla, M.; Teo, K. B. K.; Ducati, C.; Rupesinghe, N. L.; Amaratunga, G. A. J.; Ferrari, A. C.; Roy, D.; Robertson, J.; Milne, W. I. Growth Process Conditions of Vertically Aligned Carbon Nanotubes Using Plasma Enhanced Chemical Vapor Deposition. J. Appl. Phys. 2001, 90, 53085317.

66. Teo, K. B. K.; Chhowalla, M.; Amaratunga, G. A. J.; Milne, W. I.; Hasko, D. G.; Pirio, G.; Legagneux, P.; Wyczisk, F.; Pribat, D. Uniform Patterned Growth of Carbon Nanotubes without Surface Carbon. Appl. Phys. Lett. 2001, 79, 1534-1536.

67. Merkulov, V. I.; Lowndes, D. H.; Wei, Y. Y.; Eres, G. Patterned Growth of Individual and Multiple Vertically Aligned Carbon Nanofibers. Appl. Phys. Lett. 2000, 76, 3555-3557.

68. Melechko, A. V.; Merkulov, V. I.; McKnight, T. E.; Guillorn, M. A.; Klein, K. L.; Lowndes, D. H.; Simpson, M. L. Vertically Aligned Carbon Nanofibers and Related Structures: Controlled Synthesis and Directed Assembly. J. Appl. Phys. 2005, 97, 041301.

69. Cantoro, M.; Hofmann, S.; Pisana, S.; Scardaci, V.; Parvez, A.; Ducati, C.; Ferrari, A. C.; Blackburn, A. M.; Wang, K. Y.; Robertson, J. Catalytic Chemical Vapor Deposition of Single-Wall Carbon Nanotubes at Low Temperatures. Nano Lett. 2006, 6, 1107-1112.

70. Hofmann, S.; Sharma, R.; Ducati, C.; Du, G.; Mattevi, C.; Cepek, C.; Cantoro, M.; Pisana, S.; Parvez, A.; CervantesSodi, F.; et al. In Situ Observations of Catalyst Dynamics during Surface-Bound Carbon Nanotube Nucleation. Nano Lett. 2007, 7, 602-608.

71. Casiraghi, C.; Hartschuh, A.; Lidorikis, E.; Qian, H.; Harutyunyan, H.; Gokus, T.; Novoselov, K. S.; Ferrari, A. C. Rayleigh Imaging of Graphene and Graphene Layers. Nano Lett. 2007, 7, 2711-2717.

72. Nair, R. R.; Blake, P.; Grigorenko, A. N.; Novoselov, K. S.; Booth, T. J.; Stauber, T.; Peres, N. M. R.; Geim, A. K. Fine Structure Constant Defines Visual Transparency of Graphene. Science 2008, 320, 1308.

73. Blake, P.; Hill, E. W.; Castro Neto, A. H.; Novoselov, K. S.; Jiang, D.; Yang, R.; Booth, T. J.; Geim, A. K. Making Graphene Visible. Appl. Phys. Lett. 2007, 91, 063124

74. Kuzmenko, A. B.; van Heumen, E.; Carbone, F.; van der Marel, D. Universal Optical Conductance of Graphite. Phys. Rev. Lett. 2008, 100, 117401. 
75. Seepujak, A.; Bangert, U.; Harvey, A. J.; Costa, P.M.F.J.; Green, M. L. H. Redshift and Optical Anisotropy of Collective $\Pi$-Volume Modes in Multiwalled Carbon Nanotubes. Phys. Rev. B 2006, 74, 075402.

76. Stéphan, O.; Taverna, D.; Kociak, M.; Suenaga, K.; Henrard, L.; Colliex, C. Dielectric Response of Isolated Carbon Nanotubes Investigated by Spatially Resolved Electron Energy-Loss Spectroscopy: From Multiwalled to SingleWalled Nanotubes. Phys. Rev. B 2002, 66, 155422.

77. Rivacoba, A.; García de Abajo, F. J. Electron Energy Loss in Carbon Nanostructures. Phys. Rev. B 2003, 67, 085414.

78. Kociak, M.; Henrard, L.; Stéphan, O.; Suenaga, K.; Colliex, C. Plasmons in Layered Nanospheres and Nanotubes Investigated by Spatially Resolved Electron Energy-Loss Spectroscopy. Phys. Rev. B 2000, 61, 13936-13944.

79. Stökli, T.; Bonard, J.-M.; Châtelain, A.; Wang, Z. L.; Stadelmann, P. Plasmon Excitations in Graphitic Carbon Spheres. Phys. Rev. B 1998, 57, 15599-15612.

80. Stökli, T.; Bonard, J.-M.; Châtelain, A.; Wang, Z. L.; Stadelmann, P. Plasmon Excitations in Graphitic Carbon Spheres Measured by EELS. Phys. Rev. B 2000, 61, 5751-5759.

81. Lucas, A. A.; Henrard, L.; Lambin, Ph. Computation of the Ultraviolet Absorption and Electron Inelastic Scattering Cross Section of Multishell Fullerenes. Phys. Rev. B 1994, 49, 2888-2896.

82. Pichler, T.; Knupfer, M.; Golden, M. S.; Fink, J.; Cabioc'h, T. Electronic Structure and Optical Properties of ConcentricShell Fullerenes from Electron-Energy-Loss Spectroscopy in Transmission. Phys. Rev. B 2001, 63, 155415.

83. Partoens, B.; Peeters, F. M. From Graphene to Graphite: Electronic Structure around the K Point. Phys. Rev. B 2006, $74,075404$.

84. Geim, A. K.; Novoselov, K. S. The Rise of Graphene. Nat. Mater. 2007, 6, 183-191.

85. Djurisic, A. B.; Li, E. H. Optical Properties of Graphite. J. Appl. Phys. 1999, 85, 7404-7410.

86. Taft, E. A.; Philipp, H. R. Optical Properties of Graphite. Phys. Rev. 1965, 138, A197-A202.

87. Draine, B. T. Scattering by Interstellar Dust Grains. II. Xrays. Astrophys. J. 2003, 598, 1026-1037.

88. Marinopoulos, A. G.; Reining, L.; Rubio, A.; Olevano, V. Ab Initio Study of the Optical Absorption and Wave-VectorDependent Dielectric Response of Graphite. Phys. Rev. B 2004, 69, 245419.

89. Wooten, F. Optical Properties of Solids; Academic Press: New York, 1972.

90. Kunz, K. S.; Luebbers, R. J. The Finite-Difference TimeDomain Methods; CRC Press: Boca Raton, FL, 1993.

91. Lidorikis, E.; Egusa, S.; Joannopoulos, J. D. Effective Medium Properties and Photonic Crystal Superstructures of Metallic Nanoparticle Arrays. J. Appl. Phys. 2007, 101, 054304.

92. Huang, K. C.; Lidorikis, E.; Jiang, X.; Joannopoulos, J. D.; Nelson, K. A.; Bienstman, P.; Fan, S. Nature of Lossy Bloch States in Polaritonic Photonic Crystals. Phys. Rev. B 2004, 69, 195111.

93. Contopanagos, H. F.; Kyriazidou, C. A.; Merrill, W. M.; Alexópoulos, N. G. Effective Response Functions for Photonic Bandgap Materials. J. Opt. Soc. Am. A 1999, 16, 1682.

94. Ito, T.; Okazaki, S. Pushing the Limits of Lithography. Nature (London) 2000, 406, 1027-1031.

95. Service, R. F. Optical Lithography Goes to Extremes - and Beyond. Science 2001, 293, 785-786.

96. Moon, S.; Jeon, C.; Hwang, H.-N.; Hwang, C.-C.; Song, H.; Shin, H.-J.; Chung, S.; Park, C.-Y. Nanolayer Patterning Based on Surface Modification with Extreme Ultraviolet Light. Adv. Mater. 2007, 19, 1321-1324.

97. Jonkers, J. High Power Extreme Ultraviolet (EUV) Light Sources for Future Lithography. Plasma Sources Sci. Technol. 2006, 15, S8-S16.

98. Chen, J. H.; Huang, Z. P.; Wang, D. Z.; Yang, S. X.; Wen, J. G.; Ren, Z. F. Electrochemical Synthesis of Polypyrrole/Carbon Nanotube Nanoscale Composites Using Well-Aligned
Carbon Nanotube Arrays. Appl. Phys. A: Mater. Sci. Process. 2001, 73, 129-131.

99. Wilson, N. R.; Macpherson, J. V. Single-Walled Carbon Nanotubes as Templates for Nanowire Conducting Probes. Nano Lett. 2003, 3, 1365-1369.

100. Wolny, F.; Weissker, U.; Muhl, T.; Leonhardt, A.; Menzel, S.; Winkler, A.; Buchner, B. Iron-Filled Carbon Nanotubes As Probes for Magnetic Force Microscopy. J. Appl. Phys. 2008, 104, 064908.

101. Jankoviě, L.; Gournis, D.; Trikalitis, P. N.; Arfaoui, I.; Cren, T.; Rudolf, P.; Sage, M.-H.; Palstra, T. M.; Kooi, B.; De Hosson, J. T. Carbon Nanotubes Encapsulating Superconducting Single-Crystalline Tin Nanowires. Nano Lett. 2006, 6, 11311135.

102. Srinivasan, K. N.; Shanmugam, N. V.; Selvam, M.; John, S.; Shenoi, B. A. Nickel-Black Solar Absorber Coatings. Energy Convers. Manage. 1984, 24, 255-258.

103. Kodama, S.; Horiuchi, M.; Kunii, T.; Kuroda, K. Ultrablack Nickel-Phosphorus Alloy Optical Absorber. IEEE Trans. Instrum. Meas. 1990, 39, 230-232.

104. Persky, M. J. Review of Black Surfaces for Space-Borne Infrared Systems. Rev. Sci. Instrum. 1999, 70, 2193-2217.

105. Dury, M. R.; Theocharous, T.; Harrison, N.; Fox, N.; Hilton, M. Common Black Coatings-reflectance and Ageing Characteristics in the $0.32-14.3 \mu \mathrm{m}$ Wavelength Range. Opt. Commun. 2007, 270, 262-272.

106. Wang, Y. F.; Fu, W. G.; Feng, M.; Cao, X. W. Investigation of the Structure and the Physical Properties of NickelPhosphorus Ultrablack Surfaces. Appl. Phys. A: Mater. Sci. Process. 2008, 90, 549-553.

107. Huang, Y.-F.; Chattopadhyay, S.; Jen, Y.-J.; Peng, C.-Y.; Liu, T.-A.; Hsu, Y.-K.; Pan, C.-L.; Lo, H.-C.; Hsu, C.-H.; Chang, Y.-H.; Lee, C.-S.; Chen, K.-H.; Chen, L.-C. Improved Broadband and Quasi-Omnidirectional Anti-Reflection Properties with Biomimetic Silicon Nanostructures. Nat. Nanotechnol. 2007, 2, 770-774.

108. Granqvist, C. G. Solar Energy Materials. Adv. Mater. 2003, 15, 1789-1803. 